\title{
Broadband resolution analysis for imaging with measurement noise
}

\author{
Albert Fannjiang \\ Department of Mathematics, University of California, Davis, California 95616-8633, USA \\ Knut Sølna \\ Department of Mathematics, University of California, Irvine, California, USA
}

Received April 12, 2006; revised September 26, 2006; accepted October 12, 2006; posted December 8, 2006 (Doc. ID 75433); published May 9, 2007

Resolution analysis for imaging in the presence of noise is presented. A simple definition of resolution that takes into account the effect of noise is introduced and is shown to depend also on factors such as the signalto-noise ratio and the false-alarm rate. The striking effect of aperture-independent superresolution in imaging with broadband signals is demonstrated. (C) 2007 Optical Society of America

OCIS codes: $100.6640,110.4280,110.4980$.

\section{INTRODUCTION}

Consider the imaging of an object with a thin lens of $\left(z_{o}, z_{i}\right)$ geometry satisfying the lens equation $z_{o}^{-1}+z_{i}^{-1}=f^{-1}$, where $z_{o}$ and $z_{i}$ are the respective distances from the object plane and image plane to the thin lens and $f$ is the focal length of the lens. In the Fresnel diffraction theory, the image field with the object field $\Psi_{o}(\mathbf{x})$ is given by

$$
\begin{aligned}
\Psi(\mathbf{x})= & \frac{e^{i k\left(z_{o}+z_{i}\right)}}{-\lambda^{2} z_{o} z_{i}} e^{\left(i k^{\prime} / 2 z_{i}\right)|\mathbf{x}|^{2}} \\
& \times \int_{A} e^{-\left(i k / z_{i}\right) \mathbf{x} \cdot \mathbf{x}^{\prime}} \int e^{-\left(i k / z_{o}\right) \mathbf{x}^{\prime} \cdot \mathbf{x}^{\prime \prime}} \Psi_{o}\left(\mathbf{x}^{\prime \prime}\right) \mathrm{d} \mathbf{x}^{\prime \prime} \mathrm{d} \mathbf{x}^{\prime},
\end{aligned}
$$

with $\lambda$ the wavelength and $k$ the wavenumber, from which one can derive Abbé's and Rayleigh's theories of resolution. This formula is equivalent to $G \star\left[\mathrm{I}_{A} T G \star \Psi_{o}\right]$, where $\star$ stands for convolution, $G$ is the Green function (see below), the indicator function $\mathbb{I}_{A}$ stands for truncation by the aperture $A$, and $T$ is the quadratic phase factor $\exp \left\{-i k|\mathbf{x}|^{2} /(2 f)\right\} .^{1}$ What the lens does is to turn the diverging front into a converging front through the effect of the quadratic phase factor; see Fig. 1.

Another way of turning a divergent wave into a convergent wave is by using a time-reversal or phase-conjugate mirror $(\mathrm{PCM})^{2}$; see Fig. 2. A PCM replaces the incident complex wave field with its time-reversed replica and therefore reverses the direction of propagation. Consequently the phase-conjugated field can be considered as an antidistorted field, and when it retraces its path through the phase-distorting medium, the distortion is undone and refocusing on the source occurs. Mathematically the process can be expressed as $G \star\left[I_{A} G^{*} \star \Psi_{o}^{*}\right]$, where $*$ stands for conjugation. In this case, the image plane coincides with the object plane.
Both imaging processes can be written in the general form $G \star\left[\mathrm{I}_{A} U\left(G \star \Psi_{o}\right)\right]$, where $U$ is some unitary operator representing the functionality of the imaging system: In the case of a thin convex lens, $U$ is the multiplication by $T$; in the case of PCM, $U$ is the phase conjugation. Both can be interpreted as coherent measurement at the lens/ PCM followed by repropagation in the free space, which can be carried out in the physical space or the computational space. The latter perspective is particularly useful, as it permits extension of the resolution theory for optical imaging to radio-wave imaging with an antenna array.

Ambiguity is, however, present in the conventional definitions of imaging resolution. In the classical, more pessimistic Rayleigh criterion, the resolution is taken to be the radius of the first Airy disk ( $0 \%$ intensity level), while in the more optimistic Sparrow criterion, the resolution is taken to be roughly the radius of the $50 \%$ intensity level, corresponding to the minimum separation for which the midpoint intensity is not higher than that at the equal source points. Indeed, any criterion in between is an equally legitimate notion of resolution, and all are to a certain extent a measure of the size of the main lobe of the point-spread function of the imaging system. On the other hand, if noise is absent and postprocessing of the detected image is allowed, one can in principle achieve arbitrarily fine resolution from the point-spread function of the imaging system. This is known as superresolution. ${ }^{3-12}$ Indeed, the frequency components of an input image of finite extent that have not been transmitted through the band-limited imaging system may still be recovered by the technique of analytic continuation or other postprocessing methods. However, it is well known that this problem is ill-posed; i.e., small noise present in the data results in large error in the estimation. Thus resolution is limited ultimately by channel uncertainty such as imprecise measurement, due to noise, and imperfect knowledge 


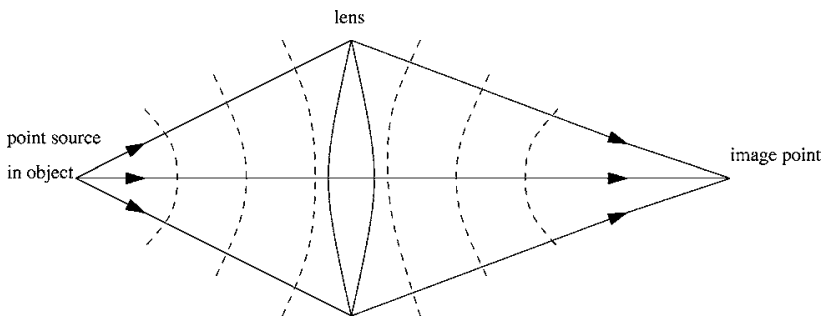

Fig. 1. Imaging with a lens.

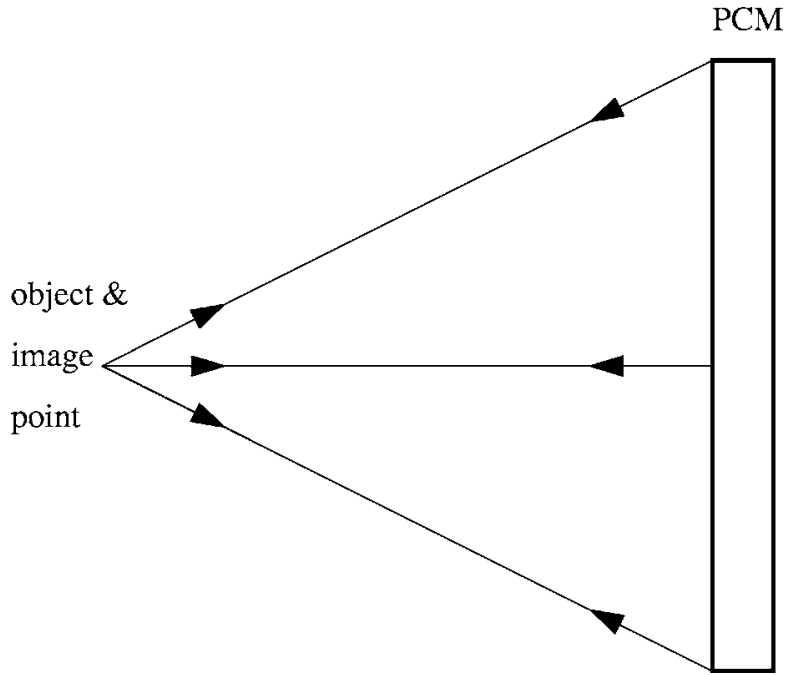

Fig. 2. Imaging with a phase-conjugate mirror (PCM).

of the imaging system. As a result the signal-to-noise ratio (SNR) in image formation should be a fundamental factor in the objective notions of resolution. ${ }^{13}$

More recently, the effect of noise on resolution has been revisited by Shahram and Milanfar, who constructed a maximum-likelihood estimator for the distance between two point sources and demonstrated numerically that resolution below the diffraction limit is attainable for sufficiently large $\mathrm{SNR}^{14}$ (see also references therein). The maximum-likelihood estimator is, however, difficult to obtain in general (such as for imaging with a broad bandwidth). Also, a precise definition of resolution taking into account SNR was not given.

We believe that a simple definition of resolution as a performance yardstick for the imaging systems of the kinds discussed above (direct imaging, instead of image reconstruction) in the presence of noise will be useful, and in this work we present such a definition and pursue some of its consequences. Roughly speaking, the new (to our knowledge) notion of resolution takes the form of the deterioration of the detection probability, given the falsealarm rate and SNR, and therefore depends on the noise ensemble as well as wavelength and aperture.

Just as in the conventional notions of resolution, the notions of resolution introduced in the present work contain arbitrariness. But the qualitative features of these asymptotic results are definitely unambiguous. Using the proposed definition of resolution, we analyze the resolution-enhancement effect with broadband signals. A most striking effect of broadband imaging is that the resulting resolution can be aperture independent, which is somewhat counterintuitive but physically sensible; see Section 3

The rest of the paper is organized as follows: In Subsection 2.A we introduce the setup of the problem with an array of transducers and an imbedded point source. Next, in Subsection 2.B we construct an imaging function and associated detection rule in the case with Gaussian measurement noise. The lateral and range resolution derive from the detection rule and are identified and analyzed in Subsections 2.C, 2.D, and 2.E. In Subsection 2.F we extend the analysis to two-point resolution. In Subsection 2.G we present a simulated example. We apply the new definition of resolution to imaging with multiple frequencies in Section 3 and then conclude in Section 4.

\section{IMAGING OF POINT SOURCE}

We work primarily with the discrete setup, which is most convenient for our approach. The discrete setup is natural in array imaging with radio waves. In imaging with lens and PCM, we consider the situation of extracting an image on the image plane by, e.g., a CCD camera which outputs a discrete array of data through the pixels. Consequently, we adopt largely the language of array imaging with the direct imaging systems described in Section 1 in mind.

In particular, we draw on the PCM imaging system for analogy, and we construct an imaging function, $\mathcal{I}(\mathbf{x})$, which corresponds to time reversing (phase conjugating) and backpropagating the received signals in the computational domain. Applying the techniques of hypothesis testing in statistics, we then derive a strategy for deciding the presence/absence of a point source based on the imaging function. The new notion of resolution is based on the outcome of the test.

\section{A. Array Imaging}

The experimental setup with an active array is shown in Fig. 3. The medium is located in the half-space $z>0$ and the transmitters array at the surface $z=0$. We consider an array of $N \times N$ receivers. The measurements at the $N^{2}$ receivers for a point source at $\overrightarrow{\mathbf{x}}_{s}$ are denoted $\mathbf{G}\left(\overrightarrow{\mathbf{x}}_{s}\right)$. This vector of observations is sometimes also referred to as the illumination vector. We consider the case with scalar waves. The time-harmonic version of the problem is then characterized by the reduced wave equation with a constant index of refraction in the situation when the back-

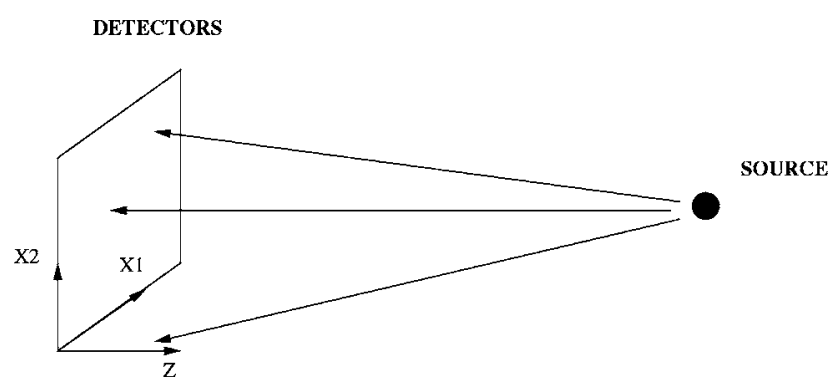

Fig. 3. Experimental setup with a passive transducer array that we consider in this paper. A source emits a signal that is recorded at the array. The two-dimensional array is located in the plane $z=0$. The cross-range space coordinates are labeled $x_{1}$ and $x_{2}$. 
ground medium is homogeneous. If we let $G_{0}$ be the freespace Green's function associated with the reduced wave equation, then we can express the illumination vector when there is no measurement noise as

$$
\boldsymbol{G}\left(\overrightarrow{\mathbf{x}}_{s}\right) \equiv\left\{G_{0}\left(\overrightarrow{\mathbf{x}}_{s}, \overrightarrow{\mathbf{x}}_{i}\right)\right\}_{i=1}^{N^{2}},
$$

where $\overrightarrow{\mathbf{x}}=(\mathbf{x}, z)=\left(x_{1}, x_{2}, z\right)$ and the free-space Green's function is given by

$$
G_{0}\left(\overrightarrow{\mathbf{x}}_{1}, \overrightarrow{\mathbf{x}}_{2}\right)=\frac{e^{i k\left|\overrightarrow{\mathbf{x}}_{1}-\overrightarrow{\mathbf{x}}_{2}\right|}}{4 \pi\left|\overrightarrow{\mathbf{x}}_{1}-\overrightarrow{\mathbf{x}}_{2}\right|},
$$

with $k=\omega / c_{0}$ the wavenumber for $c_{0}$ the wave speed and $\omega$ the temporal frequency. In the next section we discuss the case when we have additive measurement noise and use the noisy illumination vector to detect the source.

\section{B. Optimal Detection}

We consider the additive white Gaussian noise (AWGN) as present in either the intermediate stage of "coherent" measurement by the antenna array/lens/PCM or the final stage of image formation as explained in Section 1. AWGN is perhaps the simplest model representing measurement and ambient noise as well as model imperfections. As the difference between the two scenarios of introducing noise is a unitary propagation (the convolution with the freespace Green function), the final noise statistic is still the same (namely, additive white Gaussian) and therefore makes no difference to our analysis.

Therefore we assume the following model of noisy observations:

$$
\boldsymbol{Y}=\tau \boldsymbol{G}\left(\overrightarrow{\mathbf{x}}_{s}\right)+\sigma \boldsymbol{W}
$$

for the real source strength parameter being $\tau>0$ and $\boldsymbol{W}$ a complex, circularly symmetric standard Gaussian random vector: $\boldsymbol{W}=\left(\boldsymbol{W}_{r}+i \boldsymbol{W}_{i}\right)$ with $\boldsymbol{W}_{r}$ and $\boldsymbol{W}_{i}$ having identically independently distributed (i.i.d.) entries distributed according to the standard normal distribution. We seek to infer from these measurements the presence/absence of a point source and the range of uncertainty of its location.

As in the standard statistical hypothesis testing, ${ }^{15}$ we postulate two hypotheses and derive a decision rule for deciding between them based on the imaging function:

The null hypothesis $\mathcal{H}_{0}$ : The point source is absent.

The alternate hypothesis $\mathcal{H}_{1}$ : The point source is present.

Let $\alpha$ be the false-alarm rate defined as

$$
\alpha=\mathbb{P}\left[\text { accept } \mathcal{H}_{1} \mid \mathcal{H}_{0} \text { true }\right],
$$

and $1-\beta$ the detection power or probability of detection:

$$
1-\beta=\mathbb{P}\left[\text { accept } \mathcal{H}_{1} \mid \mathcal{H}_{1} \text { true }\right],
$$

with $\mathbb{P}$ representing probability. Given the data $\boldsymbol{Y}$, the decision rule for accepting $\mathcal{H}_{0}$ or not can be derived from the Neyman-Pearson lemma, which asserts that for a prescribed false-alarm rate $\alpha$, the most powerful test corresponds to accepting $\mathcal{H}_{1}$ for the likelihood ratio of $\mathcal{H}_{1}$ to $\mathcal{H}_{0}$ exceeding a threshold $T$, determined by $\alpha$.

First, we choose as the test statistic the imaging functional

$$
\mathcal{I}(\overrightarrow{\mathbf{x}})=\mathfrak{R}\left(\boldsymbol{Y}^{\dagger} \cdot \boldsymbol{G}(\overrightarrow{\mathbf{x}})\right)\|\boldsymbol{G}(\overrightarrow{\mathbf{x}})\|_{H S}^{-1},
$$

where $\mathfrak{R}$ denotes the real part. The imaging functional is constructed by using the matched filter, which optimizes the SNR. ${ }^{15}$ The choice of location $\overrightarrow{\mathbf{x}}$ is completely arbitrary as long as it lies in the computational domain and the difference between $\overrightarrow{\mathbf{x}}$ and the source location $\overrightarrow{\mathbf{x}}_{s}$ is the mismatch of the "matched" filter, which will be used to define the notion of one-point resolution below. The complex inner product $\boldsymbol{Y}^{\dagger} \cdot \boldsymbol{G}(\overrightarrow{\mathbf{x}})$ can be interpreted as time reversing and reemitting the receptive field $\boldsymbol{Y}$ into the computation domain with the Green's function; thus the coinage timereversal detection, which is particularly appropriate in the case of broadband signals (see Section 3).

Now observe that under the null hypothesis $\mathcal{I}(\overrightarrow{\mathbf{x}})$ $\sim \mathcal{N}\left(0, \sigma^{2}\right)$, while under the alternate hypothesis

$$
\mathcal{I}(\overrightarrow{\mathbf{x}}) \sim \mathcal{N}\left(\mu(\overrightarrow{\mathbf{x}}), \sigma^{2}\right), \quad \mu(\overrightarrow{\mathbf{x}})=\tau \Re\left[\boldsymbol{G}^{\dagger}\left(\overrightarrow{\mathbf{x}}_{s}\right) \cdot \boldsymbol{G}(\overrightarrow{\mathbf{x}})\right]\|\boldsymbol{G}(\overrightarrow{\mathbf{x}})\|_{2}^{-1},
$$

with $\mathcal{N}\left(\mu, \sigma^{2}\right)$ denoting the normal distribution with mean $\mu$ and standard deviation $\sigma$. As mentioned, the NeymanPearson lemma corresponds to accepting $\mathcal{H}_{1}$ for the likelihood ratio exceeding a specific threshold $T$. Here, the likelihood ratio is the ratio of the two probability densities of the imaging function $\mathcal{I}$ that corresponds to $\mathcal{H}_{1}$ and $\mathcal{H}_{2}$, respectively, and when evaluated at the observation $\mathcal{I}(\overrightarrow{\mathbf{x}})$. Using the expression for the normal density, we find that the likelihood ratio is given by

$$
\Lambda(\overrightarrow{\mathbf{x}})=C \exp \left(\Re\left[\boldsymbol{Y}^{\dagger} \cdot \boldsymbol{G}(\overrightarrow{\mathbf{x}})\right]\|\boldsymbol{G}(\overrightarrow{\mathbf{x}})\|_{2}^{-1} \mu(\overrightarrow{\mathbf{x}}) \sigma^{-2}\right),
$$

where $C$ is a constant depending only on $\boldsymbol{G}$.

By the Neyman-Pearson lemma, the decision rule of accepting $\mathcal{H}_{1}$ iff $\mathcal{I}(\overrightarrow{\mathbf{x}})>T$ maximizes the probability of detection for a given false-alarm rate $\alpha$ with the threshold $T$

$$
T=\sigma \Phi^{-1}(1-\alpha),
$$

where $\Phi$ is the (Gauss) error function. Indeed, since the imaging functional is Gaussian with standard deviation $\sigma$ under $\mathcal{H}_{0}$, this definition of $T$ means that the probability of accepting $\mathcal{H}_{1}$ given that $\mathcal{H}_{0}$ is true is $\alpha$ as specified in Eq. (2).

\section{One-Point Resolution}

We now discuss the notion of one-point resolution, or uncertainty of location, as another performance criterion. This is introduced as the mismatch of the "matched" filter, resulting in a certain prescribed degree of performance deterioration.

First, let us derive a duality relation between the falsealarm rate $\alpha$ and the miss probability $\beta$. For simplicity of notation we consider the point source located at $\overrightarrow{\mathbf{x}}_{s}$ $=(\mathbf{0}, L)$. If the source is present, the imaging functional is Gaussian with mean $\mu(\overrightarrow{\mathbf{x}})$ and standard deviation $\sigma$. From this we find the power of the test, $1-\beta(\overrightarrow{\mathbf{x}})$, to be

$$
1-\beta(\overrightarrow{\mathbf{x}})=1-\Phi((T-\mu(\overrightarrow{\mathbf{x}})) / \sigma),
$$

which can be expressed in terms of the SNR

$$
\operatorname{SNR}(\overrightarrow{\mathbf{x}})=\frac{\mathbb{E}^{2}[\mathcal{I}(\overrightarrow{\mathbf{x}})]}{\operatorname{Var}[\mathcal{I}(\overrightarrow{\mathbf{x}})]}=\frac{\mu(\overrightarrow{\mathbf{x}})^{2}}{\sigma^{2}},
$$

and Eq. (5) as 


$$
\begin{aligned}
1-\beta(\overrightarrow{\mathbf{x}}) & =1-\Phi\left(\Phi^{-1}(1-\alpha)-\frac{\mu(\overrightarrow{\mathbf{x}})}{\sigma}\right) \\
& =\Phi\left(\sqrt{\operatorname{SNR}(\overrightarrow{\mathbf{x}})}-\Phi^{-1}(1-\alpha)\right),
\end{aligned}
$$

where we used the relation $\Phi(x)=1-\Phi(-x)$. We thus arrive at the following performance duality relations:

$$
\begin{array}{r}
1-\alpha=\Phi\left(\sqrt{\operatorname{SNR}(\overrightarrow{\mathbf{x}})}-\Phi^{-1}(1-\beta(\overrightarrow{\mathbf{x}}))\right), \\
1-\beta(\overrightarrow{\mathbf{x}})=\Phi\left(\sqrt{\operatorname{SNR}(\overrightarrow{\mathbf{x}})}-\Phi^{-1}(1-\alpha)\right) .
\end{array}
$$

Note that $\mu(\overrightarrow{\mathbf{x}})$ and $\operatorname{SNR}(\overrightarrow{\mathbf{x}})$ achieve the maximum at $\overrightarrow{\mathbf{x}}$ $=\overrightarrow{\mathbf{x}}_{S}$ with

$$
\operatorname{SNR}\left(\overrightarrow{\mathbf{x}}_{s}\right)=\tau^{2} N^{2} /(\sigma 4 \pi L)^{2} .
$$

Thus the detection power $1-\beta(\overrightarrow{\mathbf{x}})$ also achieves the maximum at $\overrightarrow{\mathbf{x}}=\overrightarrow{\mathbf{x}}_{s}$. Figure 4 shows the maximal detection power $1-\beta\left(\overrightarrow{\mathbf{x}}_{s}\right)$ as a function of false-alarm rate $\alpha$ at various levels of SNR. We see a trade-off between detection power and false-alarm rate. Figure 5 shows the detection power $1-\beta(\overrightarrow{\mathbf{x}})$ as a function of the relative offset parameter $\|\overrightarrow{\mathbf{x}}\| A /(\lambda L)$ for $\alpha=0.05$ and $\lambda L / A^{2}=10$ and $\operatorname{SNR}(0)$ $=2,4,6,8,10$. For large offsets the detection power approaches $\alpha$, since then the point source has little effect on the measurements. For a small offset a large SNR gives a higher detection power, as this corresponds to a relatively small additive noise in the measurements. We have used the paraxial approximation (13) for plotting Fig. 5, and here $\lambda$ is wavelength, $A$ is the aperture of the mirror, and $\lambda L / A$ is the Rayleigh cross-range resolution.

\section{Cross-Range Resolution}

Let us consider the imaging functional $\mathcal{I}(\overrightarrow{\mathbf{x}})$ at $\overrightarrow{\mathbf{x}}-(\mathbf{x}, L)$ with the offset $\mathbf{x}=\left(x_{1}, x_{2}\right)$ and ask the following question: How far off axis must the test point $\overrightarrow{\mathbf{x}}$ be moved in order to

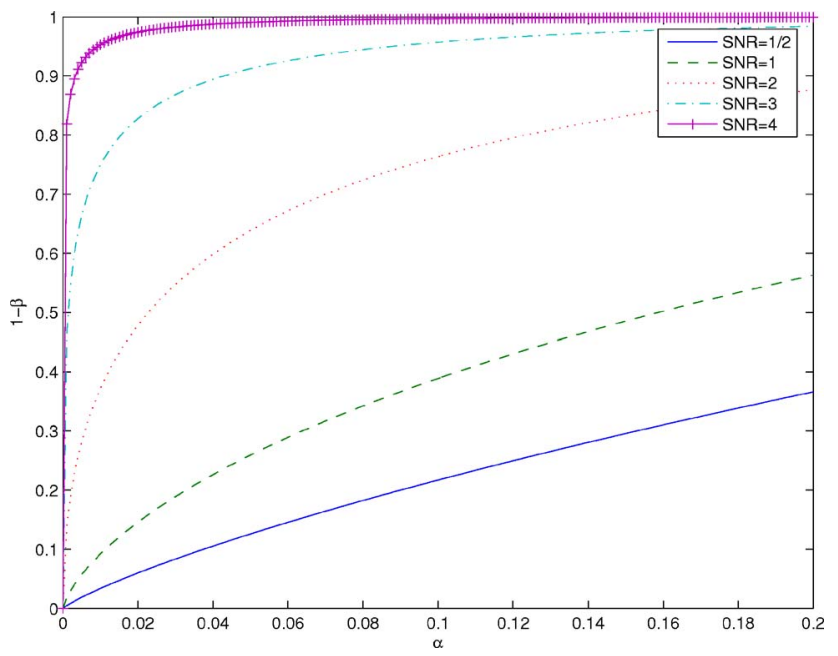

Fig. 4. (Color online) Detection power as function of $\alpha$ for $\sqrt{\operatorname{SNR}(0)} \in\{1 / 2,1,2,3,4\}$. For a small value of $\alpha$, corresponding to a small false-alarm rate, the test also has a relatively small power $1-\beta$. This follows since for small $\alpha$ the test must be conservative and conclude that the point source is present only for relatively large values of the imaging functional; see Eqs. (5) and (6). For a fixed false-alarm rate, the power increases with the SNR, since this corresponds to an increase in the signal received from the source relative to the noise.

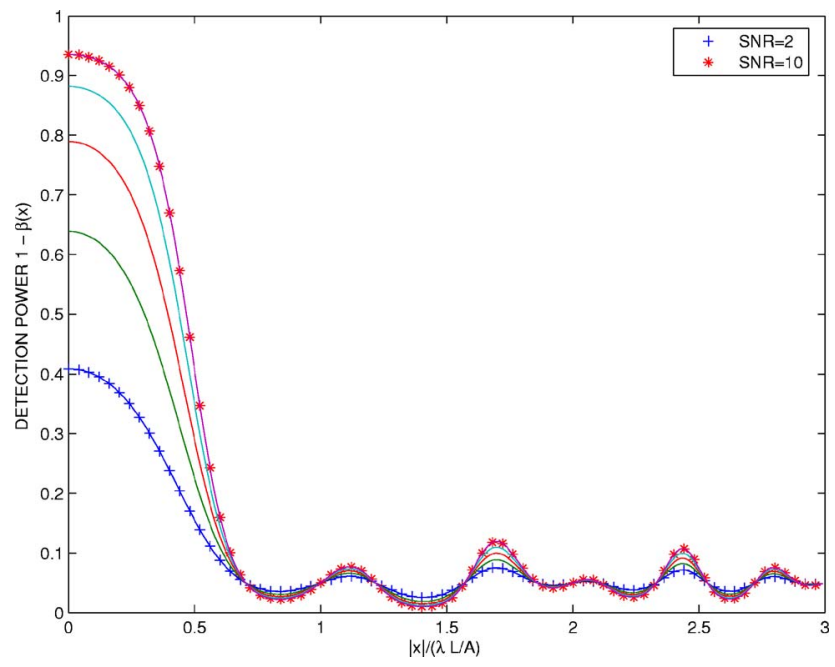

Fig. 5. (Color online) Detection power as function of offset and SNR. Here $\lambda$ is wavelength, $L$ is distance to target, and $A$ is the aperture of the mirror. As the offset increases, corresponding to increased error in the cross-range specification, the probability of detecting the source decreases.

increase the probability of failed detection over the minimal $\beta_{0}=\beta\left(\overrightarrow{\mathbf{x}}_{s}\right)$ by a specific factor $f>1$ ?

That is, $f \beta_{0}=\beta\left(\rho_{c}\right)$, where $\rho_{c}$ is the cross-range resolution $\rho_{c}$ for the given factor $f>1$ and $\beta\left(\rho_{c}\right)$ is the probability of failed detection with the offset $\rho_{c}$. The number of this criterion is, however, generally more than one, and we define the resolution to be the largest root. The cross-range resolution can be interpreted as the uncertainty of the source location due to the presence of noise and the sensitivity of the detection scheme characterized by $f$.

The factor $f>1$ is somewhat arbitrary. A reasonable choice is such that $f \beta_{0}$ is exactly the midpoint between the minimum $\beta_{0}$, at the target location, and the maximum at infinity. At infinity, SNR is zero and hence $\beta(\infty)$ is $1-\alpha$ by Eq. (9). With this choice,

$$
f=\frac{1}{2}+\frac{1-\alpha}{2 \beta_{0}}
$$

Other choices of $f$ are fine as long as they satisfy the following constraint:

$$
1<f \leq \frac{1-\alpha}{\beta_{0}} .
$$

From Eq. (11) and (12) we see that for a fixed falsealarm rate, $f$ must decrease as SNR decreases since $\beta_{0}$ would increase in this case. In fact, this notion of resolution can be thought of as a generalized Sparrow resolution in the presence of noise in the sense that with $f=2$, the miss probability at the midpoint of two incoherent point sources separated by $\rho_{c}$ is roughly equal to that at either source point.

For simplicity we now consider the paraxial approximation of the Green's function (1)

$$
G_{0}\left(\left(x_{1}, x_{2}, 0\right),(\rho, 0, L)\right) \approx \frac{1}{4 \pi L} \exp \left[i k\left(L+\frac{\left|x_{1}-\rho\right|^{2}+\left|x_{2}\right|^{2}}{2 L}\right)\right],
$$

provided that 


$$
\rho \ll L, \quad A \ll L .
$$

It then follows from Eqs. (4) and (7) that

$$
\sqrt{\operatorname{SNR}(\rho)} \approx \frac{\tau}{4 \pi L \sigma} \mathfrak{R}\left(e^{-i k \rho^{2} /(2 L)} \sum_{j=1}^{N} e^{i k x_{j} \rho / L}\right),
$$

where the square-array elements $\mathbf{x}_{i j}=\left(x_{i}, x_{j}\right)$ are assumed to be equally spaced with $x_{1}=-A / 2 \ldots x_{N}=A / 2$. We further assume the number of elements is sufficiently large so that

$$
\rho / N \ll R_{c},
$$

where $R_{c}=\lambda L / A, \lambda=2 \pi / k$, is Abbe's (or Rayleigh's) crossrange resolution. Then, using Eq. (15),

$$
\begin{aligned}
\sqrt{\operatorname{SNR}(\rho)} & \approx \frac{\tau}{\sigma(4 \pi L)} \cos \left(\frac{\pi \rho^{2}}{\lambda L}\right) \frac{N}{A} \int_{-A / 2}^{A / 2} e^{-i k y \rho / L} \mathrm{~d} y \\
& =\sqrt{\operatorname{SNR}(0)} \cos \left(\frac{\pi \rho^{2}}{\lambda L}\right) \operatorname{sinc}\left(\frac{\pi \rho}{R_{c}}\right) .
\end{aligned}
$$

Recall that

$$
\begin{gathered}
\sqrt{\operatorname{SNR}\left(\rho_{c}\right)}=\Phi^{-1}(1-\alpha)+\Phi^{-1}\left(1-f \beta_{0}\right), \\
\sqrt{\operatorname{SNR}(0)}=\Phi^{-1}(1-\alpha)+\Phi^{-1}\left(1-\beta_{0}\right),
\end{gathered}
$$

and we deduce the equation determining the cross-range resolution

$$
\sqrt{\frac{\operatorname{SNR}\left(\rho_{c}\right)}{\operatorname{SNR}(0)}}=\cos \left(\frac{\pi \rho_{c}^{2}}{\lambda L}\right) \operatorname{sinc}\left(\frac{\pi \rho_{c}}{R_{c}}\right)=\frac{1}{F\left(\alpha, \beta_{0}\right)},
$$

with

$$
F\left(\alpha, \beta_{0}\right)=\frac{\Phi^{-1}(1-\alpha)+\Phi^{-1}\left(1-\beta_{0}\right)}{\Phi^{-1}(1-\alpha)+\Phi^{-1}\left(1-f \beta_{0}\right)} .
$$

We define the resolution gain by $g_{c}=R_{c} / \rho_{c}$. Condition (16) then becomes $g_{c} \gg 1 / N$. Since the resolution is defined as the largest root of Eq. (20), the cos factor in Eq. (20) can be neglected.

Figure 6 shows the cross-range resolution gain $g_{c}$ as a function of SNR and for $\alpha=0.001,0.005,0.01,0.02,0.05$. For each value of $\alpha$ in the plot, the lower cutoff in the SNR value corresponds to constraint (12). The resolution gain increases with SNR and is always greater than one. For a fixed SNR, the resolution gain increases with the falsealarm rate, which reflects the trade-off between detection power and false-alarm rate seen in Fig. 4. Figure 7 shows the cross-range resolution gain $g_{c}$ as a function of the detection power $1-\beta_{0}$ with $f=2$ and for $\alpha=0.001,0.005$, $0.01,0.02,0.05$. The resolution gain increases with the detection probability. For a fixed detection probability, the resolution gain decreases with the false-alarm rate since this corresponds to a decreased SNR.

To understand how SNR affects the detection resolution, let us derive an asymptotic formula for the crossrange resolution as $\operatorname{SNR}\left(\overrightarrow{\mathbf{x}}_{s}\right)$ tends to infinity. In this regime, $\beta_{0} \rightarrow 0$ and

$$
\frac{1}{F} \approx \frac{\Phi^{-1}\left(1-f \beta_{0}\right)}{\Phi^{-1}\left(1-\beta_{0}\right)} \approx \sqrt{1+\frac{\ln f}{\ln \beta_{0}}},
$$

following from the asymptotic

$$
1-\Phi(t) \sim \frac{1}{t \sqrt{2 \pi}} e^{-t^{2} / 2}, \quad t \gg 1 .
$$

Comparing the Taylor expansions of $\operatorname{sinc}\left(\pi \rho_{c} / R_{c}\right)$ and the right-hand side of Eq. (22), we obtain

$$
\rho_{c} \sim \frac{L}{A k} \sqrt{\frac{12 \ln f}{-\ln \beta_{0}}} \sim \frac{L}{A k} \sqrt{\frac{24 \ln j}{\operatorname{SNR}(0)}}
$$

and, equivalently,

$$
g_{c} \sim \pi \sqrt{\frac{\operatorname{SNR}(0)}{6 \ln f}} .
$$

We see that the resolution gain increases like the square root of SNR, and therefore superresolution (i.e., $g_{c}>1$ )

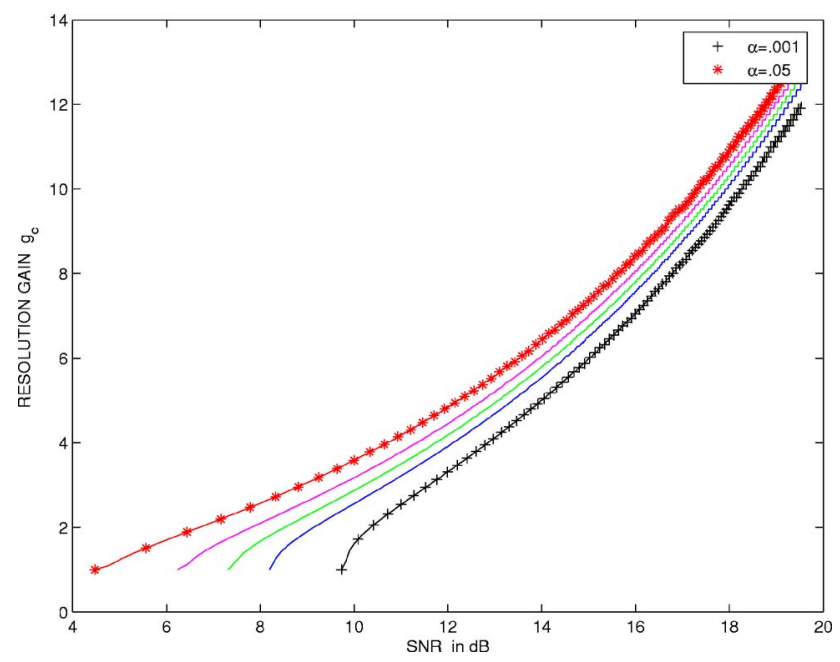

Fig. 6. (Color online) Cross-range resolution gain $g_{c}$ as function of $\operatorname{SNR}(0)$ and $\alpha$ with $f=2$.

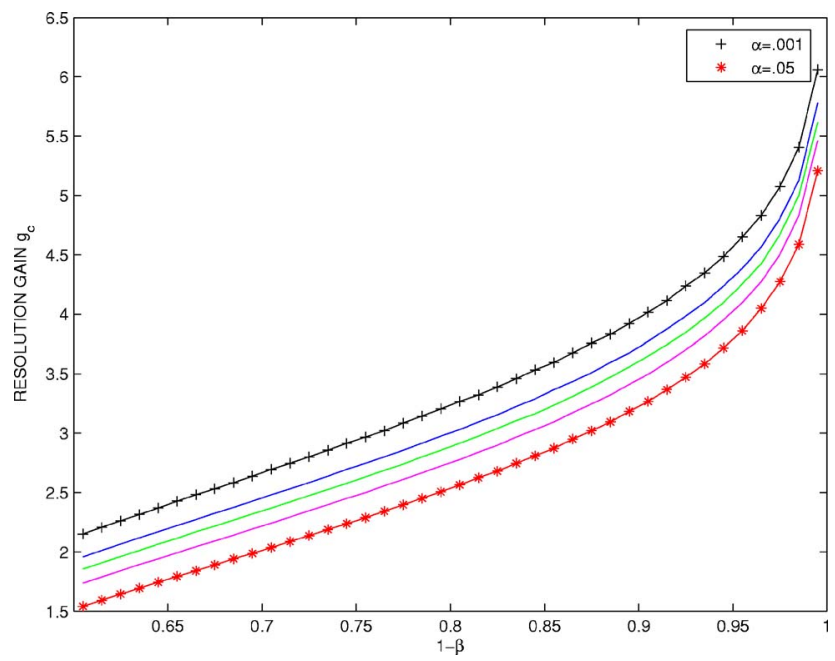

Fig. 7. (Color online) Cross-range resolution gain $g_{c}$ as function of the detection power $1-\beta_{0}$ and $\alpha$ with $f=2$. 


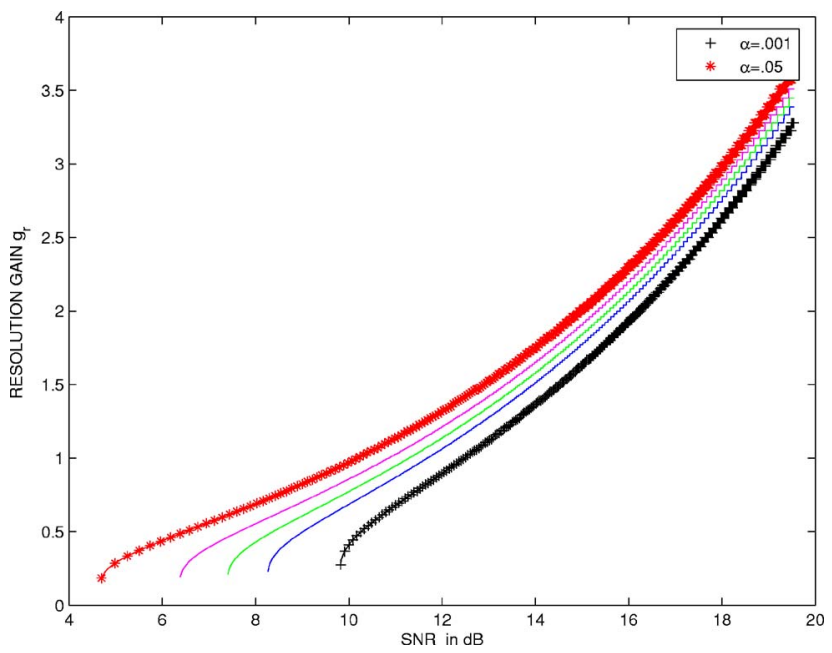

Fig. 8. (Color online) Range resolution gain $g_{r}$ as a function of $\operatorname{SNR}(0)$ and $\alpha$ with $f=2$.

can be achieved with sufficiently high SNR for any given $f>1$.

\section{E. Range Resolution}

The notion of resolution can be extended to the offset along the axis between the array and the source point. Analogous to the cross-range resolution, the range resolution $\rho_{r}$ is determined by the equation $f \beta(0)=\beta\left(\rho_{r}\right)$, with some prescribed $f>1$.

Using Eqs. (7) and (13) in the paraxial regime, we have

$$
\sqrt{\frac{\operatorname{SNR}\left(\rho_{r}\right)}{\operatorname{SNR}(0)}}=\frac{1}{N^{2}} \Re\left(e^{-i k \rho_{r}} \sum_{l, m=1}^{N} e^{-i k \rho_{r}\left[\left(x_{1}^{2}+x_{m}^{2}\right) / 2 L^{2}\right]}\right) .
$$

In the absence of noise the Rayleigh criterion for range resolution is $R_{r}=\lambda L^{2} / A^{2}$. The range resolution gain $g_{r}$ $=R_{r} / \rho_{r}$ is determined by the following analog of Eq. (20):

$$
\begin{aligned}
\sqrt{\frac{\operatorname{SNR}\left(\rho_{r}\right)}{\operatorname{SNR}(0)}} & \approx \cos \left(k R_{r} / g_{r}\right)\left(\int_{-1 / 2}^{1 / 2} \cos \left(\pi x^{2} / g r\right) \mathrm{d} x\right)^{2} \\
& \approx \frac{1}{F\left(\alpha, \beta_{0}\right)} .
\end{aligned}
$$

The asymptotic for the range resolution gain $g_{r}$ at high SNR can now be derived as before:

$$
g_{r} \sim \pi \sqrt{\frac{\operatorname{SNR}(0)}{80 \ln f}} \text { for } \operatorname{SNR}(0) \rightarrow \infty .
$$

Figure 8 shows the range resolution gain as function of SNR and the false-alarm rate for $\alpha=0.001,0.005,0.01$, $0.02,0.05$. In contrast to the cross-range resolution, the range resolution gain may go below one as SNR decreases. This is an important observation, which also illustrates how incorporating the notion of noise provides additional perspectives on classical resolution measures. In the range direction, single-frequency imaging gives poor resolution, and in fact with strong noise even the pessimistic Rayleigh criterion for resolution may be too optimistic. In Section 3 we shall see that this picture changes in the broadband case.

\section{F. Two-Point Resolution}

In this section, we analyze the resolution of two incoherent point sources in the presence of noise. In the absence of noise, the classical Sparrow criterion says that two point sources of equal intensity cannot be resolved if and only if the midpoint intensity does not dip. This criterion is no longer appropriate in the presence of noise since the noise may significantly enhance the midpoint intensity above the intensity at the locations of the sources at the separation of the Sparrow criterion.

Let us consider the following signal model for two incoherent point sources of identical intensity:

$$
\boldsymbol{Y}=\tau\left(e^{-i \pi \theta} \boldsymbol{G}_{1}+e^{i \pi \theta} \boldsymbol{G}_{2}\right)+\sigma \boldsymbol{W},
$$

where $\theta$ is the random phase uniformly distributed in $[-1 / 2,1 / 2]$ and $\boldsymbol{G}_{1}, \boldsymbol{G}_{2}$ are the illumination vectors from the first and second source points, respectively:

$$
\boldsymbol{G}_{1} \equiv\left\{G_{0}\left(\overrightarrow{\mathbf{x}}_{s_{1}}, \overrightarrow{\mathbf{x}}_{i}\right)\right\}_{i=1}^{N^{2}}, \quad \boldsymbol{G}_{2} \equiv\left\{G_{0}\left(\overrightarrow{\mathbf{x}}_{s_{2}}, \overrightarrow{\mathbf{x}}_{i}\right)\right\}_{i=1}^{N^{2}},
$$

for $\overrightarrow{\mathbf{x}}_{s_{1}}, \overrightarrow{\mathbf{x}}_{s_{2}}$, the location of the two sources. We assume that the noise $\boldsymbol{W}$ and the random phase variable $\theta$ are independent. We further assume that the two point sources are located at $\overrightarrow{\mathbf{x}}_{s_{1}}=(-\rho, 0, L), \overrightarrow{\mathbf{x}}_{s_{2}}=(\rho, 0, L)$ for the discussion of cross-range resolution and $\overrightarrow{\mathbf{x}}_{s_{1}}=(0,0, L-\rho), \overrightarrow{\mathbf{x}}_{s_{2}}$ $=(0,0, L+\rho)$ for the discussion of range resolution. For simplicity of presentation, we again restrict ourselves to the paraxial approximation.

We consider an imaging function of the same form as before $\mathcal{I}(\overrightarrow{\mathbf{x}})=\mathfrak{R}\left[\boldsymbol{Y}^{\dagger} \cdot \boldsymbol{G}(\overrightarrow{\mathbf{x}})\right]\|\boldsymbol{G}(\overrightarrow{\mathbf{x}})\|_{2}^{-1}$, which has mean with respect to the noise $\boldsymbol{W}: \tau \Re\left[e^{i \pi \theta} \boldsymbol{G}_{1}^{\dagger} \cdot \boldsymbol{G}(\overrightarrow{\mathbf{x}})\right.$ $\left.+e_{1}^{-i \pi \theta} \boldsymbol{G}_{2}^{\dagger} \cdot \boldsymbol{G}(\overrightarrow{\mathbf{x}})\right]\|\boldsymbol{G}(\overrightarrow{\mathbf{x}})\|_{2}^{-1}$. Following the classical Sparrow resolution criterion in the noiseless case, we consider the imaging function $\mathcal{I}\left(\overrightarrow{\mathbf{x}}_{0}\right)$ at the midpoint $\overrightarrow{\mathbf{x}}_{0}=(0,0, L)$ of the two point sources as well as at the source points $\overrightarrow{\mathbf{x}}_{s_{1}}, \overrightarrow{\mathbf{x}}_{s_{2}}$. We write $\boldsymbol{G}_{0}=\boldsymbol{G}(0,0, L)$.

We consider the cross-range resolution. In the paraxial approximation, the imaging function at the midpoint

$$
\begin{aligned}
\mathcal{I}\left(\overrightarrow{\mathbf{x}}_{0}\right) \approx & \frac{N_{\tau}}{4 \pi L} 2 \cos (\pi \theta) \cos \left(\frac{\pi \rho^{2}}{\lambda L}\right) \operatorname{sinc}\left(\frac{\pi \rho}{R_{c}}\right)+\sigma \Re\left[\boldsymbol{W}^{\dagger} \cdot \boldsymbol{G}_{0}\right] \\
& \times\left\|\boldsymbol{G}_{0}\right\|_{2}^{-1}
\end{aligned}
$$

has the mean

$$
\mathbb{E} \mathcal{I}\left(\overrightarrow{\mathbf{x}}_{0}\right) \approx \frac{N_{\tau}}{\pi^{2} L} \mathcal{F}_{c}(\rho), \quad \mathcal{F}_{c}(\rho) \equiv \cos \left(\frac{\pi \rho^{2}}{\lambda L}\right) \operatorname{sinc}\left(\frac{\pi \rho}{R_{c}}\right) .
$$

The imaging function at the source points $\overrightarrow{\mathbf{x}}_{s_{i}}, i=1,2$,

$$
\mathcal{I}\left(\overrightarrow{\mathbf{x}}_{s_{i}}\right) \approx \frac{N_{\tau}}{4 \pi L} \cos (\pi \theta)\left(1+\mathcal{F}_{c}(2 \rho)\right)+\sigma \Re\left[\boldsymbol{W}^{\dagger} \cdot \boldsymbol{G}_{i}\right]\left\|\boldsymbol{G}_{i}\right\|_{2}^{-1}
$$

has the mean

$$
\mathbb{E} \mathcal{I}\left(\overrightarrow{\mathbf{x}}_{i}\right) \approx \frac{\tau N}{2 \pi^{2} L}\left(1+\mathcal{F}_{c}(2 \rho)\right), \quad i=1,2 .
$$

Note that the random variable $x=\cos (\pi \theta)$ has the Chebyshev density 


$$
h(x)=\frac{2}{\pi \sqrt{1-x^{2}}}, \quad x \in(0,1),
$$

with the mean $2 / \pi$ and the variance $\left(\pi^{2}-8\right) /\left(2 \pi^{2}\right)$. Thus the fluctuation of $\mathcal{I}\left(\overrightarrow{\mathbf{x}}_{s_{i}}\right), i=0,1,2$ has the probability density function (pdf) $\psi$ given by the convolution of the Gaussian and the centered Chebyshev pdf's after proper normalization.

In the noiseless case the classical Sparrow resolution $\rho_{s}$ can be reformulated as $\mathbb{E} \mathcal{I}\left(\overrightarrow{\mathbf{x}}_{0}\right)=\mathbb{E} \mathcal{I}\left(\overrightarrow{\mathbf{x}}_{s_{i}}\right), i=1,2$, i.e.,

$$
1+\mathcal{F}_{c}(2 \rho)=2 \mathcal{F}_{c}(\rho) .
$$

In the presence of noise, we need to consider fluctuation and noise as well as the mean.

For the noisy case we need to consider the signal-tofluctuation ratio (SFR) at the test points $\overrightarrow{\mathbf{x}}_{0}, \overrightarrow{\mathbf{x}}_{s_{i}}, i=1,2$, defined as

$$
\operatorname{SFR}\left(\overrightarrow{\mathbf{x}}_{s_{i}}\right)=\frac{\left|\mathbb{E} \mathcal{I}\left(\overrightarrow{\mathbf{x}}_{s_{i}}\right)\right|^{2}}{\operatorname{Var}\left(\mathcal{I}\left(\overrightarrow{\mathbf{x}}_{s_{i}}\right)\right)}=\frac{4 / \pi^{2}}{1 / 2-4 / \pi^{2}+\left(\operatorname{SNR}\left(\overrightarrow{\mathbf{x}}_{s_{i}}\right)\right)^{-1}} .
$$

In the relatively noisy case with

$$
\mathrm{SNR} \ll \frac{2 \pi^{2}}{\pi^{2}-8} \approx 17.3,
$$

we have $\mathrm{SFR} \approx 4 \times \mathrm{SNR} / \pi^{2}$. Under such conditions, the measurement noise dominates over the incoherent fluctuation of the source, and we may assume the distribution of the imaging functional is Gaussian. Here we are deciding between two alternatives:

The null hypothesis $\mathcal{H}_{1}$ : The source is one point of strength $2 \tau$ at zero offset.

The alternate hypothesis $\mathcal{H}_{2}$ : The source is two points of equal strength $\tau$.

We use the imaging function as the basis for our decision. Under $\mathcal{H}_{1}$ we have $\mathcal{I}\left(\overrightarrow{\mathbf{x}}_{0}\right) \sim \mathcal{N}\left(\mu_{1}, \sigma\right)$, while under $\mathcal{H}_{2}$, $\mathcal{I}\left(\overrightarrow{\mathbf{x}}_{0}\right) \sim \mathcal{N}\left(\mu_{2}, \sigma\right)$ with

$$
\begin{aligned}
& \mu_{1}=\frac{N_{\tau}}{\pi^{2} L}, \\
& \mu_{2}=\frac{N_{\tau}}{\pi^{2} L} \mathcal{F}_{c}(\rho) .
\end{aligned}
$$

Let $\alpha$ be the probability of accepting $\mathcal{H}_{2}$ while $\mathcal{H}_{1}$ is correct and $\beta$ be the probability of accepting $\mathcal{H}_{1}$ while $\mathcal{H}_{2}$ is correct. Note that $\alpha$ is independent of $\rho$ but $\beta$ is clearly a function of $\rho$. Since $\tau>0, \mu_{i} \geq 0$ and for a given $\alpha$, the decision rule is to accept $\mathcal{H}_{2}$ when $\mathcal{I}$ goes below a certain threshold and vice versa. The threshold is determined by

$$
T=\mu_{1}+\sigma \Phi^{-1}(\alpha)
$$

which is independent of $\rho$. This is important as the detection rule can then be used even when the parameter $\rho$ is unknown.

The detection probability for a two-point source is then given by

$$
1-\beta(\rho)=\Phi\left(\frac{T-\mu_{2}(\rho)}{\sigma}\right)=\Phi\left(\Phi^{-1}(\alpha)+\frac{\mu_{1}-\mu_{2}(\rho)}{\sigma}\right)
$$

or equivalently

$$
\Phi^{-1}(1-\beta(\rho))=\Phi^{-1}(\alpha)+\frac{\mu_{1}-\mu_{2}(\rho)}{\sigma} .
$$

According to the Neyman-Pearson lemma, the detector is the most powerful in the sense that it produces the highest detection probability for all values of the unknown parameter $\rho$ and a given false-alarm rate.

We may define the detection resolution as the offset that gives a $50 \%$ (or any value between $\beta_{0}$ and 99\%) chance of detecting the presence of two source points, that is, $\beta\left(\rho_{c}\right)=1 / 2$. This then gives

$$
\mu_{2}\left(\rho_{c}\right)=\mu_{1}+\sigma \Phi^{-1}(\alpha)
$$

which we write in the form

$$
\cos \left(\frac{\pi \rho_{c}^{2}}{\lambda L}\right) \operatorname{sinc}\left(\frac{\pi \rho_{c}}{R_{c}}\right)=1+\frac{\sigma \Phi^{-1}(\alpha)}{N \tau /\left(\pi^{2} L\right)} \equiv 1+\frac{\pi \Phi^{-1}(\alpha)}{4 \sqrt{\operatorname{SNR}(0)}}
$$

As commented before, the cos factor in the above equation can be dropped and the resolution gain $g_{c}=R_{c} / \rho_{c}$ can be determined from the equation

$$
\operatorname{sinc}\left(\frac{\pi}{g_{c}}\right)=1+\frac{\pi \Phi^{-1}(\alpha)}{4 \sqrt{\operatorname{SNR}(0)}} .
$$

Figure 9 shows the resolution gain as a function of the SNR, that is, $\sqrt{\operatorname{SNR}(0)}$ for $\alpha=0.001,0.005,0.01,0.02$, 0.05 . For a fixed SNR the resolution gain again increases with the false-alarm rate.

Considering the regime where $\left|\Phi^{-1}(\alpha)\right| \ll \sqrt{\operatorname{SNR}(0)}$ and expanding the left-hand side of the equation in the Taylor series, we obtain

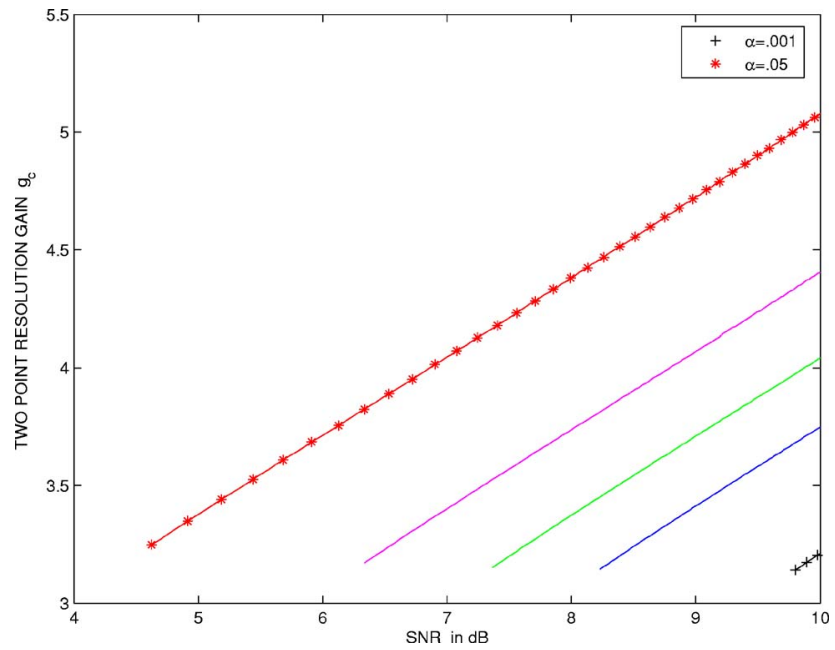

Fig. 9. (Color online) Two-point cross-range resolution gain $g_{c}$. 


$$
g_{c} \approx \sqrt{\frac{2 \pi}{3}} \frac{\operatorname{SNR}(0)^{1 / 4}}{\left|\Phi^{-1}(\alpha)\right|^{1 / 2}}
$$

The two-point resolution gain depends on SNR in a way different from the one-point resolution gain [Eq. (23)]; with uncertainty about two rather than one location, the relative enhancement in resolution as a function of SNR becomes weaker. The validity of Eq. (33) is constrained by Eq. (27). In the high-SNR limit, the random phase difference dominates the SFR $(\approx 7)$ and is the ultimate limitation to two-point resolution with the measurement noise playing no role.

We finish this section by briefly commenting on the two-point range resolution. The null and alternative hypotheses are as above, now with $\overrightarrow{\mathbf{x}}_{s_{1}}=(0,0, L-\rho)$ and $\overrightarrow{\mathbf{x}}_{s_{2}}$ $=(0,0, L+\rho)$. We can then repeat the analysis presented above in the cross-range resolution case. The only modification in our analysis arises in the computation of $\mu_{2}$ in Eq. (29) and corresponds to

$$
\mathcal{F}_{c}(\rho) \mapsto \mathcal{F}_{r}(\rho)=\cos (k \rho)\left(\int_{-1 / 2}^{1 / 2} \cos \left(\pi x^{2} \rho / R_{r}\right) \mathrm{d} x\right)^{2} .
$$

Thus, in view of the calculation leading to Eq. (24), the range resolution is now determined by the following modification of Eq. (32):

$$
\left(\int_{-1 / 2}^{1 / 2} \cos \left(\pi x^{2} / g_{r}\right) \mathrm{d} x\right)^{2}=1+\frac{\pi \Phi^{-1}(\alpha)}{4 \sqrt{\operatorname{SNR}(0)}},
$$

with $g_{r}=R_{r} / \rho$. Thus the two-point resolution scales with the Rayleigh range resolution $R_{r}$.

Considering the regime where $\left|\Phi^{-1}(\alpha)\right| \ll \sqrt{\operatorname{SNR}(0)}$ and expanding again in Taylor series, we obtain the asymptotics

$$
g_{r} \approx \sqrt{\frac{\pi}{20}} \frac{\operatorname{SNR}(0)^{1 / 4}}{\left|\Phi^{-1}(\alpha)\right|^{1 / 2}}
$$

\section{G. Illustration of One-Point Resolution}

In this section we consider a simple example using the detection rule

$$
\mathcal{I}(\overrightarrow{\mathbf{x}})>T
$$

to create an image. The point source is located at the origin, and we use the parameters: $L=100, A=10, \tau=100$, $\sigma=1, N=12, k=2$, and $\alpha=0.05$. Note that in this case $\mathrm{SNR} \approx 4$. For the simulation we use the exact Green's function, rather than its parabolic approximation, and the Monte Carlo method. The performance as a function of relative offset is shown in Fig. 10. Comparing Figs. 10 and 5, we find a slightly better performance in Fig. 10 than in the theoretical prediction in Fig. 5 by using the paraxial approximation. With the same parameters as in Fig. 10, Fig. 11 depicts the profile of the detection probability as a function of both range and cross-range offsets. The scales are in the units of the Rayleigh cross-range and range resolutions.

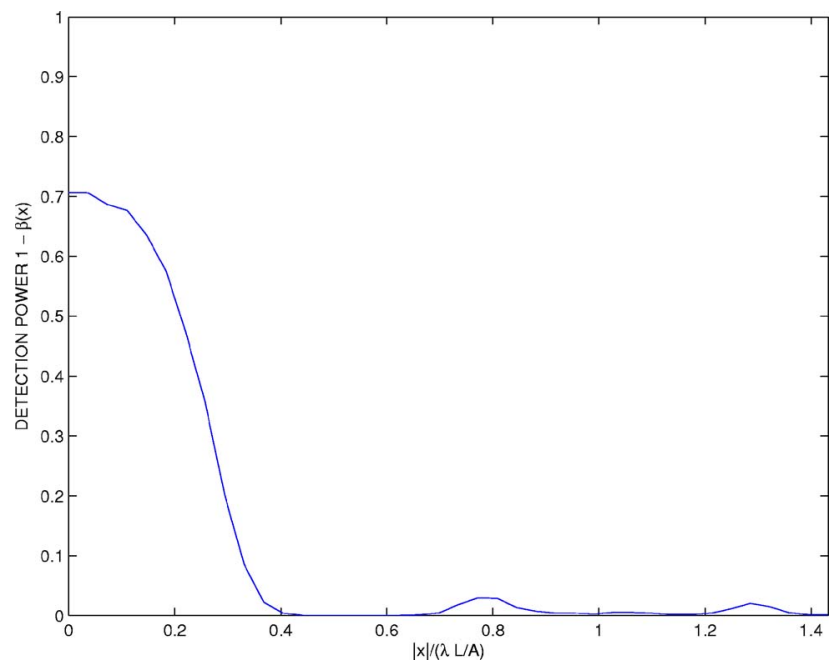

Fig. 10. (Color online) Simulated detection probability as function of cross-range offset.

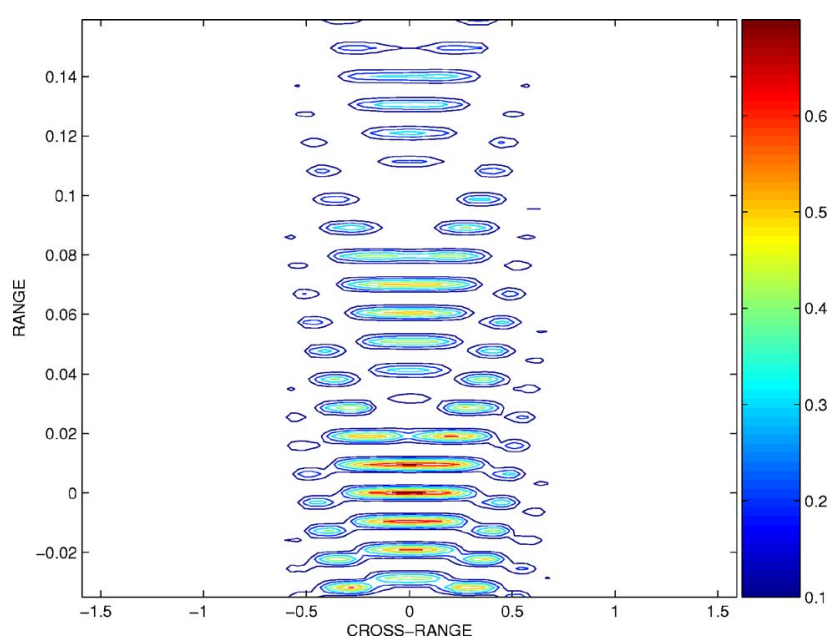

Fig. 11. (Color online) Simulated detection probability.

\section{BROADBAND IMAGING}

Performance of imaging and detection in the presence of noise may be strongly enhanced by using signals at multiple frequencies. First, the multiple frequencies provide travel-time information and improve the range resolution in particular. Second, the different frequencies may be weakly correlated and therefore provide independent information about the target. Let us analyze the performance of multifrequency imaging using the new definition of resolution.

We assume the following model of noisy measurement at wavenumber $k_{j}$ :

$$
\boldsymbol{Y}\left(k_{j}\right)=\tau \boldsymbol{G}\left(k_{j}\right)+\sigma \boldsymbol{W}_{j}, \quad j \in\{1, \ldots, W\},
$$

with $\boldsymbol{W}_{j}$ a complex (independ) Gaussian noise vector. We consider the imaging functional

$$
\mathcal{I}(\mathbf{x})=\sum_{j} \Re\left(\boldsymbol{Y}\left(k_{j}\right)^{\dagger} \cdot \boldsymbol{G}\left(\overrightarrow{\mathbf{x}} ; k_{j}\right)\right)\left\|\boldsymbol{G}\left(\overrightarrow{\mathbf{x}} ; k_{j}\right)\right\|_{2}^{-1} .
$$

The most powerful test for a given false-alarm rate $\alpha$ corresponds to rejecting $\mathcal{H}_{0}$ iff $\mathcal{I}(\overrightarrow{\mathbf{x}})>T$ with the threshold $T=\sigma \Phi^{-1}(1-\alpha)$ as before. 
For simplicity we assume that the discrete wavenumbers are evenly spaced in the interval $\{\bar{k}-\Delta k / 2, \bar{k}+\Delta k / 2\}$ with the spacing $\delta k=\Delta k /(W-1)$. Note first that the detection power achieves the maximum $1-\beta_{0}$ at the location $\overrightarrow{\mathbf{x}}=\overrightarrow{\mathbf{x}}_{s}$ :

$$
1-\beta_{0}=\Phi\left(\sqrt{\mathrm{W} \operatorname{SNR}\left(\overrightarrow{\mathbf{x}}_{s}\right)}-\Phi^{-1}(1-\alpha)\right),
$$

with the same $\operatorname{SNR}\left(\overrightarrow{\mathbf{x}}_{s}\right)$ as in Eq. (10). Therefore, the multiple frequencies enhance the detection performance via higher SNR.

\section{A. Cross-Range Resolution}

As before, we analyze the multifrequency cross-range resolution in the paraxial approximation. The offset dependent SNR in Eq. (15) now becomes

$$
\sqrt{\operatorname{SNR}(\rho)}=\frac{\tau}{\sigma \sqrt{W} 4 \pi L} \sum_{j=1}^{W} \Re\left(e^{-i k_{j} \rho^{2} /(2 L)} \sum_{l=1}^{N} e^{i k_{j} x_{1} \rho / L}\right) .
$$

The cross-range resolution $\rho_{f, k}$ in the multifrequency case is determined by the equation

$$
\begin{aligned}
\frac{1}{F\left(\alpha, \beta_{o}\right)} & =\sqrt{\frac{\operatorname{SNR}(\rho)}{\operatorname{SNR}(0)}} \\
& =\frac{1}{W N} \sum_{j=1}^{W} \sum_{l=1}^{N} \cos \left(-i k_{j} \frac{\rho^{2}-2 \rho x_{l}}{2 L}\right) \\
& =\frac{1}{N} \sum_{l=1}^{N} \Re\left[e^{-i k_{1} y l} \frac{1-e^{-i W \delta k y_{l}}}{W\left(1-e^{-i \delta k y l}\right)}\right],
\end{aligned}
$$

with

$$
y_{l}=\frac{\rho^{2}-2 \rho x_{l}}{2 L} .
$$

To understand explicitly how multiple frequencies can enhance resolution, we analyze two particular regimes. First, let us consider the narrowband regime:

$$
\Delta k\left(\rho^{2}+\rho A\right) / L \ll 1,
$$

which turns out to be equivalent to the conventional definition $\Delta k \ll \bar{k}$. Under the narrowband condition, we obtain from Eq. (37) that

$$
\begin{aligned}
\sqrt{\frac{\operatorname{SNR}(\rho)}{\operatorname{SNR}(0)}} & \approx \frac{1}{N} \sum_{l=1}^{N} \cos \left[\frac{\bar{k}\left(\rho^{2}-2 \rho x_{l}\right)}{2 L}\right] \\
& \approx \cos \left(\frac{\bar{k} \rho^{2}}{2 L}\right) \operatorname{sinc}\left(\frac{\bar{k} A \rho}{2 L}\right) .
\end{aligned}
$$

In other words, the narrowband case is approximately the same as the one-frequency case.

Consider next the broadband case $\bar{k} \sim \Delta k$, or equivalently

$$
\Delta k \rho^{2} / L=O(1),
$$

with a small aperture $A \ll \rho$. This implies that $y_{l}$ $\approx \rho^{2} /(2 L)$. Then as $\delta k \rightarrow 0$ and $W \rightarrow \infty$, we obtain from Eq. (37) that

$$
\begin{aligned}
\frac{1}{F\left(\alpha, \beta_{0}\right)} & \approx \frac{2 L}{\rho^{2} \Delta k} \Re\left[i e^{-i\left(\rho^{2} / 2 L\right)(\bar{k}+\Delta k / 2)}-i e^{-i\left(\rho^{2} / 2 L\right)(\bar{k}-\Delta k / 2)}\right] \\
& =\cos \left(\frac{\pi \rho^{2}}{\bar{\lambda} L}\right) \operatorname{sinc}\left(\frac{\rho^{2} \Delta k}{4 L}\right), \quad \bar{\lambda}=\frac{2 \pi}{\bar{k}},
\end{aligned}
$$

which leads to the multifrequency cross-range resolution determined by

$$
\frac{1}{F\left(\alpha, \beta_{0}\right)}=\cos \left(2 \pi \frac{\lambda_{B}}{\bar{\lambda}}\left(\frac{\rho}{\sqrt{2 \lambda_{B} L}}\right)^{2}\right) \operatorname{sinc}\left(\pi\left(\frac{\rho}{\sqrt{2 \lambda_{B} L}}\right)^{2}\right),
$$

with the modulation wavelength $\lambda_{B}=2 \pi / \Delta k$. Therefore, $\rho=O\left(\sqrt{\lambda_{B} L}\right)$ and can be arbitrarily small in the high-SNR limit. Note that this result is independent of the aperture $A$. Thus, we have compensated a small aperture with bandwidth so that the cross-range resolution is on the scale of the Fresnel length $\sqrt{\lambda_{B} L}$ of the modulation.

\section{B. Range Resolution}

Again we consider the paraxial regime with $A \ll L$ so that

$$
\begin{aligned}
\frac{1}{F\left(\alpha, \beta_{0}\right)} & =\sqrt{\frac{\operatorname{SNR}\left(\rho_{r}\right)}{\operatorname{SNR}(0)}} \\
& =\frac{1}{W N^{2}} \Re\left(\sum_{j=1}^{W} \sum_{l, m=1}^{N} e^{-i k_{j} \rho_{r}} e^{-i k_{j} \rho_{r}\left(x_{l}^{2}+x_{m}^{2}\right) / 2 L^{2}}\right) \\
& \approx \frac{1}{W} \Re\left(\sum_{j=1}^{W} e^{-i k_{j} \rho_{r}}\right),
\end{aligned}
$$

which determines the range resolution $\rho_{r}$. Equivalently we have

$$
\frac{1}{F\left(\alpha, \beta_{0}\right)}=\cos \left(\frac{2 \pi \lambda_{B} \rho_{r}}{\bar{\lambda} \lambda_{B}}\right) \operatorname{sinc}\left(\frac{\pi \rho_{r}}{\lambda_{B}}\right)
$$

for $\lambda_{B}=2 \pi / \Delta k$. Therefore, $\rho_{r}=O\left(\lambda_{B}\right)$ and can be reduced indefinitely in the high-SNR limit. This result is again independent of the aperture.

\section{Two-Point Resolution}

We can easily generalize the two-point resolution analysis of Subsection 2.F to the multifrequency case. The derivation of the gain is completely analogous modulo two replacements, as we describe next. The only modifications in the analysis arise in the computation of $\mu_{1}$ and $\mu_{2}$ in Eqs. (28) and (29); the modifications arise since the imaging function now is given by Eq. (36) with $W>1$. We consider the relatively noisy broadband case with small aperture $A \ll \rho$, as discussed above for the single-frequency case. The computation leading to Eqs. (39) and (40) then show that the generalization to the multifrequency case follows from the replacements: (i) $\tau \mapsto \sqrt{W} \tau$, reflecting an enhanced SNR; (ii) $\mathcal{F}_{c}(\rho) \mapsto \mathcal{F}_{c, k}(\rho)$ and $\mathcal{F}_{r}(\rho) \mapsto \mathcal{F}_{r, k}(\rho)$ for cross-range and range resolution, respectively, where 


$$
\begin{aligned}
& \mathcal{F}_{c, k}(\rho)=\cos \left(2 \pi \frac{\lambda_{B}}{\bar{\lambda}}\left(\frac{\rho}{\sqrt{2 \lambda_{B} L}}\right)^{2}\right) \operatorname{sinc}\left(\pi\left(\frac{\rho}{\sqrt{2 \lambda_{B} L}}\right)^{2}\right), \\
& \mathcal{F}_{r, k}(\rho)=\cos \left(2 \pi \frac{\lambda_{B}}{\bar{\lambda}}\left(\frac{\rho_{r}}{\lambda_{B}}\right)\right) \operatorname{sinc}\left(\pi\left(\frac{\rho_{r}}{\lambda_{B}}\right)\right)
\end{aligned}
$$

see Eqs. (39) and (40).

\section{CONCLUSIONS}

We have presented the performance analysis for direct imaging in the presence of noise by introducing a simple notion of resolution. We have analyzed one-point and twopoint resolution in the framework of statistical hypothesis testing.

For a fixed false-alarm rate, the resolution gain increases with SNR and bandwidth. In the case with high SNR or large bandwidth, the resolution is typically much better than the Abbé (or Rayleigh) resolution. We have demonstrated a striking effect of broadband imaging, namely, aperture-independent superresolution.

We plan to extend our approach to the case of broadband imaging in a random medium, which amounts to multiplicative noise.

\section{ACKNOWLEDGMENTS}

Albert Fannjiang was supported by Office of Naval Research (ONR) grant N00014-02-1-0090, DARPA grant N00014-02-1-0603, and National Science Foundation (NSF) grant DMS 0306659. Knut Sølna was supported by ONR grant N00014-02-1-0090, Darpa grant N00014-02-10603, NSF grant DMS 0307011, and the Sloan Foundation.
Corresponding author Knut Sølna can be reached by e-mail at ksolna@math.uci.edu; Albert Fannjiang's e-mail address is fannjiang@math.ucdavis.edu.

\section{REFERENCES}

1. M. Born and W. Wolf, Principles of Optics, 7th (expanded) ed. (Cambridge U. Press, 1999).

2. B. Y. Zel'dovich, N. F. Pilipetsky, and V. V. Shkunov, Principles of Phase Conjugation (Springer-Verlag, 1985).

3. M. Bendinnelli, A. Consortini, L. Ronchi, and B. R. Frieden, "Degrees of freedom and eigenfunctions for the noisy image," J. Opt. Soc. Am. 64, 1498-1502 (1974).

4. A. J. den Dekker and A. van den Bos, "Resolution: a survey," J. Opt. Soc. Am. A 14, 547-557 (1997).

5. M. A. Fiddy and T. J. Hall, "Nonuniqueness of superresolution techniques applied to sampled data," J. Opt. Soc. Am. 71, 1406-1407 (1981).

6. B. R. Frieden, "On arbitrarily perfect imagery with a finite aperture," Opt. Acta 16, 795-807 (1969).

7. R. W. Gerchberg, "Superresolution through error energy reduction," Opt. Acta 21, 709-720 (1974).

8. C. K. Rushforth, A. E. Crawford, and Y. Zhou, "Leastsquares reconstruction of objects with missing highfrequency components,” J. Opt. Soc. Am. 72, 204-211 (1982).

9. C. K. Rushforth and R. W. Harris, "Restoration, resolution and noise," J. Opt. Soc. Am. 58, 539-545 (1968).

10. B. Saleh, "A priori information and the degrees of freedom of noise images," J. Opt. Soc. Am. 67, 71-76 (1977).

11. G. Toraldo di Francia, "Resolving power and information," J. Opt. Soc. Am. 45, 497-501 (1955).

12. N. J. Vershad, "Resolution, optical-channel capacity and information theory," J. Opt. Soc. Am. 59, 157-163 (1969).

13. J. W. Goodman, Statistical Optics (Wiley, 1985).

14. M. Shahram and P. Milanfar, "Imaging below the diffraction limit: a statistical analysis," IEEE Trans. Image Process. 13, 677-689 (2004).

15. S. M. Kay, Fundamentals of Statistical Signal Processing, Detection Theory (Prentice Hall, 1998). 